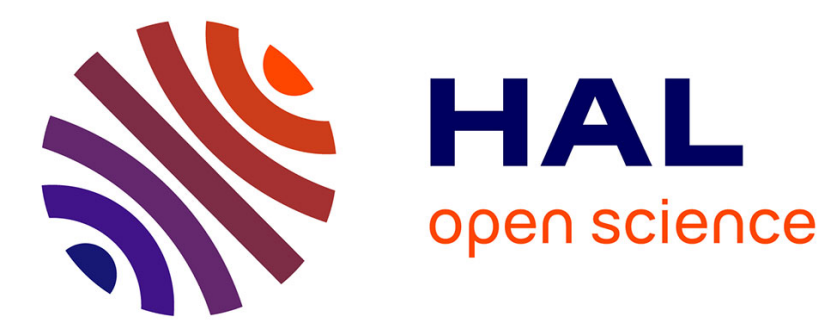

\title{
Creating and modulating rhythms by controlling the physics of the body
}

Alexandre Pitti, Ruima Niiyama, Yasuo Kuniyoshi

\section{To cite this version:}

Alexandre Pitti, Ruima Niiyama, Yasuo Kuniyoshi. Creating and modulating rhythms by controlling the physics of the body. Autonomous Robots, 2010, 28 (3), pp.317-329. 10.1007/s10514-009-9176-1 . hal-00628932

\section{HAL Id: hal-00628932 \\ https://hal.science/hal-00628932}

Submitted on 5 Oct 2011

HAL is a multi-disciplinary open access archive for the deposit and dissemination of scientific research documents, whether they are published or not. The documents may come from teaching and research institutions in France or abroad, or from public or private research centers.
L'archive ouverte pluridisciplinaire HAL, est destinée au dépôt et à la diffusion de documents scientifiques de niveau recherche, publiés ou non, émanant des établissements d'enseignement et de recherche français ou étrangers, des laboratoires publics ou privés. 


\section{ISSN 0929-5593, Volume 28, Number 3}

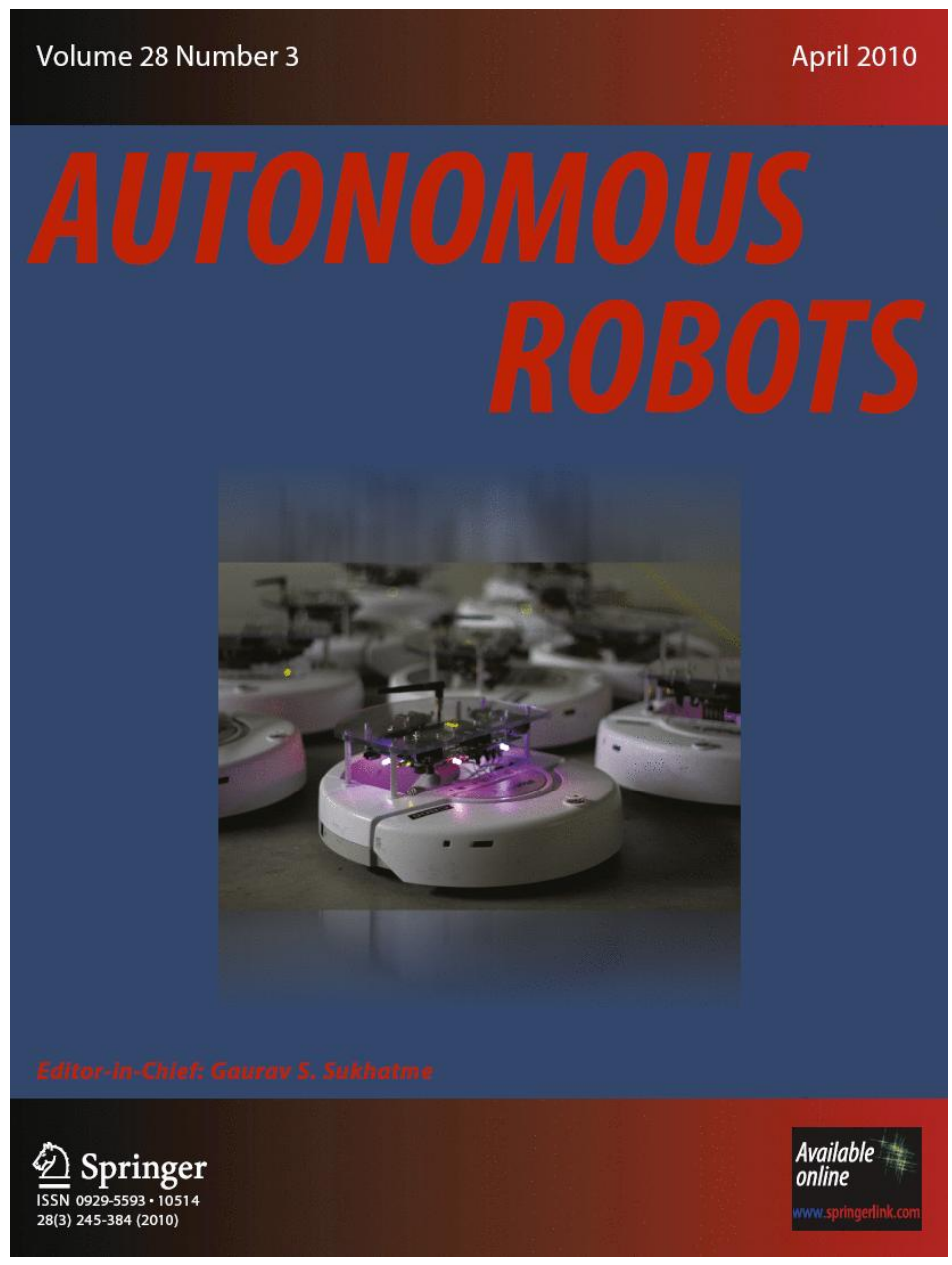

This article was published in the above mentioned Springer issue.

The material, including all portions thereof, is protected by copyright; all rights are held exclusively by Springer Science + Business Media.

The material is for personal use only;

commercial use is not permitted.

Unauthorized reproduction, transfer and/or use

may be a violation of criminal as well as civil law. 


\title{
Creating and modulating rhythms by controlling the physics of the body
}

\author{
Alexandre Pitti · Ryuma Niiyama · Yasuo Kuniyoshi
}

Received: 31 March 2009 / Accepted: 21 December 2009 / Published online: 7 January 2010

(C) Springer Science+Business Media, LLC 2010

\begin{abstract}
The motion behaviors of vertebrates require the correct coordination of the muscles and of the body limbs even for the most stereotyped ones like the rhythmical patterns. It means that the neural circuits have to share some part of the control with the material properties and the body morphology in order to rise any of these motor synergies. To this respect, the chemical downward neuromodulators that supervise the pattern generators in the spinal cord create the conditions to merge (or to disrupt) them by matching the phase of the neural controllers to the body dynamics. In this paper, we replicate this control based on phase synchronization to implement neuromodulators and investigate the interplay between control, morphology and material. We employ this mechanism to control three robotic setups of gradual complexity and actuated by McKibben type air muscles: a single air muscle, an elbow-like system and a leg-like articulation. We show that for specific values, the control parameters modulate the internal dynamics to match those of the body and of the material physics to either the rhythmical and non-rhythmical gait patterns.
\end{abstract}

Keywords Neuromodulators · Motor synergies $\cdot$ Phase synchronization $\cdot$ McKibben actuators

\footnotetext{
A. Pitti $(\bowtie)$

Eng. Bldg. 2, 7-3-1, Hongo, Bunkyo-ku, Tokyo 113-8656, Japan e-mail: alex@isi.imi.i.u-tokyo.ac.jp

R. Niiyama $\cdot$ Y. Kuniyoshi

Laboratory for Intelligent Systems and Informatics, Department of Mechano-Informatics Graduate School of Information Science and Technology, University of Tokyo, Tokyo, Japan
}

\section{Introduction}

Performing complex behaviors and rapid movements require the partial overlap between the controllers and the body's dynamics. It means that one efficient controller should encompass some knowledge about the system to be controlled (e.g., its passive dynamics). At the physical level, it implies the exploitation of its material properties (i.e., its stiffness and elasticity, the shock absorption, friction and gravity) whereas at the morphological level, it implies the exploitation of its structural regularities (i.e., its postural configuration or the disposition of its effectors). The interplay of both levels create at the body interface the conditions to rise the natural coordinations of the muscles into behavioral patterns.

In biological systems, it is the micro-circuits in the spinal cord that rule out these motor synergies (cf., Bizzi et al. 1995; Grillner 1996). However, rather than producing stereotyped rhythms as they were previously thought, they are considered nowadays to be far more flexible systems that can exploit the body dynamics instead of guiding them (cf., Bizzi and Clarac 1999; Rosenblum et al. 1998; Selverston et al. 2000; Rabinovich et al. 2006). For instance, they can adapt their rhythms to the body's ongoing motion via proprioceptive feedback (see Grillner 2006; Levi et al. 2005; Ivanenko et al. 2005; Lockhart and Ting 2007) and follow multiple synergies at the same time and/or dismiss others (cf. Ting and MacPherson 2005; Ting 2007). This suggests that they exploit efficiently the body rather than model its dynamics and that they are part of a larger distributed system that includes the body and higher-level signals which accurately controls them (Thelen and Smith 1995). One important actor of this larger system is perhaps the chemical neuromodulators which work at the intermediate level and that constantly supervise the pattern generators and tune their frequencies 
to the good synergies (Fellous and Linster 1998; Calabrese 1995; Marder and Calabrese 1996; Marder 1996). The neuromodulators, with respect to the pattern generators, create therefore the conditions to realize a specific motion. As a result, they can modulate the coordination between the neural circuits and the body dynamics.

So far, the emphasis on pattern generators in robotics to produce rhythmical patterns (see Ijspeert 2008 for a review) has a little occluded the functional role of the neuromodulators to group softly different motor synergies (Kelso 1995; Kelso and Haken 1995). Methods corresponding to the former case model entrainment by extracting the periodic and rhythmic patterns so that the specific waveforms are learned; e.g., the adaptive phase frequency technique by Ijspeert and colleagues (Ijspeert et al. 2003), and also (Schaal 2003; Buchli et al. 2006; Nakanishi et al. 2004). Conversely, those corresponding to the latter case create entrainment by exploiting the body dynamics; e.g., the morphological computation by Pfeifer and colleagues (Iida and Pfeifer 2004; cf. also, Williamson 1998; Lungarella and Berthouze 2002, 2004; Iida and Pfeifer 2004; Der et al. 2006; Kuniyoshi and Suzuki 2004; Kuniyoshi and Sangawa 2006).

In this paper, we employ a control framework based on phase synchronization to implement neuromodulators that can regulate the coordination between the body and the controllers' dynamics to different gait patterns either oscillatory or discrete. Although this approach is not novel and some similar results have been found in earlier works, we would like to investigate in detail the interplay between control, morphology and materials and its links with biological control and especially neuromodulation. We want also to extend some of our previous works realized on computer simulations to robotic systems (cf., Pitti et al. 2005, 2006; Lungarella et al. 2007). We employ for this McKibbentype pneumatic actuators that reproduce some of the nonlinear properties of mammal's muscles in terms of damping and elasticity (cf., Klute et al. 1999; Niiyama et al. 2007; Takuma and Hosoda 2007; Hosoda et al. 2008).

The paper is organized as follows. In the first part, we present the phenomenon known as phase synchronization between coupled dynamical systems. We explain how nonlinear controllers can exploit this mechanism to control some dissipative systems (i.e., the robot's passive dynamics). In the experimental part, we describe the way we use this control scheme in three robotic setups and experiments of increasing complexity. The first experiment is done with a single pneumatic muscle on which the variations of the coupling parameters modulate entrainment to its intrinsic frequency. The second and third experiments are done respectively on a 1 DOF elbow-like mechanical system actuated in the sagittal plane and a 3 DOF leg-like articulation (hip, knee and heel). Our experimental results show that variations of the coupling parameters in the systems without explicit coupling between the controllers generate dynamical transition of actuator's output behaviors and entrainment synchronized to the robot's body's natural frequency. They rise rhythmical behaviors (oscillatory regimes) as well as other gait patterns (discrete motions such as contraction and release). We discuss then the possible links with downward neuromodulators to reduce the computational complexity of motion control to few order parameters. The set of synergies created can be learned then and combined to perform more complex and fast coordinations by exploiting the material properties and the body morphology.

\section{Control framework}

In the last decade, the discoveries of control and synchronization of chaos shed some new lights on the mechanisms of interaction between nonlinear dynamical systems and complex systems in general. Pecora and Caroll demonstrated that weakly coupled chaotic oscillators can synchronize their phase to each other while their amplitude stay uncorrelated (see Pecora and Carroll 1990; Pikovsky et al. 1997; Gonzalez-Miranda 2004).

The important result is that small actions can have strong effects on the system's response to another order magnitude. It stresses the idea that physically coupled systems can efficiently interact with very little information exchanged as they exploit their passive dynamics reciprocally. In biological control, we suggest that neuromodulators may play a similar role to increase and control the computational complexity of neural circuits with a very weak input trigger.

\subsection{Mechanism of phase synchronization}

We describe in this section the mechanism of phase synchronization for the case of two coupled dynamical systems and for the more general case of multiple interacting subsystems. Then, we explain how chaotic systems can serve to the control of mechanical systems (e.g., robotic devices).

One can conceive the coupling of two oscillators $\phi_{1}$ and $\phi_{2}$ linked by the equations:

$\left\{\begin{array}{l}\dot{\phi_{1}}=\omega_{1}+\gamma_{1} g_{1}\left(\phi_{1}, \phi_{2}\right), \\ \dot{\phi_{2}}=\omega_{2}+\gamma_{2} g_{2}\left(\phi_{2}, \phi_{1}\right),\end{array}\right.$

where the pair $\left\{\gamma_{1}, \gamma_{2}\right\}$ corresponds to the coupling parameters regulating the interaction between the two systems and the functions $g_{1}$ and $g_{2}$ model the kind of interaction between the two systems (cf. Rosenblum et al. 2001; Pikovsky et al. 1997). Each oscillator perturbs the dynamics of the other as a weak and periodic perturbation force $\mathbf{P}(t)$ of frequency $\omega$ and coupling strength $\gamma$ and phase $\phi$ : $\mathbf{P}(t)=\gamma \cos (\omega t+\phi)$. Under these conditions, the two systems essentially influence each other if their frequencies are 
in resonance:

$n \omega_{1} \approx m \omega_{2}$

where $n$ and $m$ are integers. The generalized phase difference, $\varphi_{n, m}(t)$, between the two systems is then,

$\varphi_{n, m}(t)=n \phi_{1}(t)-m \phi_{2}(t)$

When the phases of the two systems stay close enough then the phase difference $\varphi_{n, m}(t)$ is kept bounded. Under this condition, it is possible to observe the phenomenon known as phase synchronization (Rosenblum et al. 1996; Pikovsky et al. 2001) and (3) becomes:

$\left|n \phi_{1}(t)-m \phi_{2}(t)\right|<\epsilon$.

Phase synchronization (PS) means that the phase of one oscillator always stays close enough to the phase of the other oscillator for some conditional pairs $\{m, n\}$. Therefore, discovering all the pairs $\{m, n\}$ that realize PS will permit to find the different types of coordination existing between the two systems. Similarly, we can expand this assumption to the synchronization of multiple forces in presence-e.g., a distributed system of multiple elements. The perturbation force $\mathbf{P}$ is then a linear combination of $k$ forces $p_{i}$ where $i \in[1, \ldots, k]: \mathbf{P}=\sum_{i=1}^{k} p_{i}$. The conditions to satisfy PS are ensured if the phases $\phi_{p_{i}}(t)$ of all interacting forces stay bounded to each other below a certain constant $\epsilon$ :

$$
\begin{array}{r}
\left\langle\left|n_{i, j} \phi_{p_{i}}(t)-m_{i, j} \phi_{p_{j}}(t)\right|\right\rangle<\epsilon, \quad \forall i \neq j, \\
\left\langle\left|\Delta \varphi_{p_{i}, p_{j}}\right|\right\rangle<\epsilon, \quad \forall i \neq j
\end{array}
$$

with \langle\rangle the mean function. The global stability of PS is satisfied if the phase relations for all the sub-systems stay always circumscribed.

If the coupling terms $\left\{\gamma_{1}, \gamma_{2}\right\}$ are also the control parameters that regulate PS for this case, then the modulation of PS will depend on the dimensionality of the coupling terms and not on the system's dimensionality per se which can be higher. We suggest that downward neuromodulators may play a similar role to the coupling parameters to tune in the micro-circuits to the body dynamics and to indirectly control the overall coordination. By doing so, they may perform a dimensionality reduction by supervising the global dynamics rather than controlling individually each sub-system.

\subsection{Joint mechanisms of phase synchronization and} feedback resonance between active and dissipative systems

A mechanical system is not properly an oscillator and the condition required to realize PS (normally restricted to oscillatory systems) is that one oscillator can exploit its non-

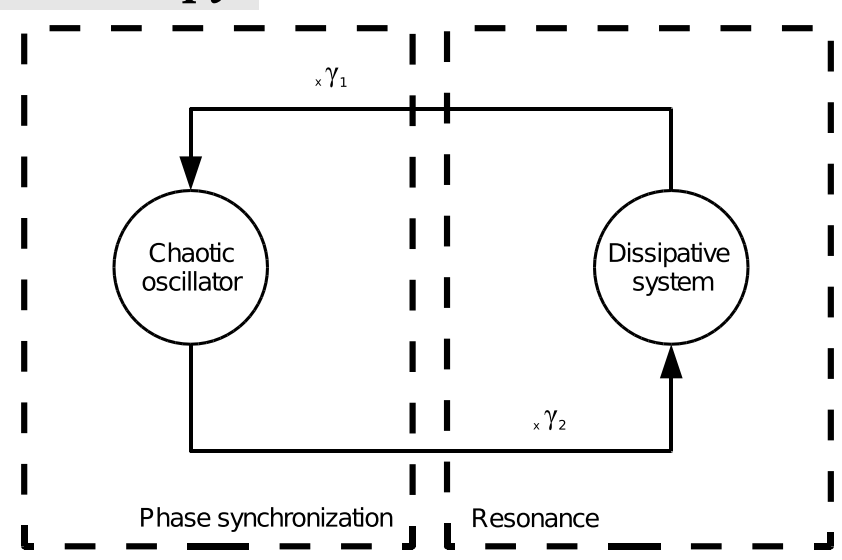

Fig. 1 Joint mechanism of phase synchronization and feedback resonance between an active system and a dissipative system. Depending on the values of the coupling parameter $\left\{\gamma_{1}, \gamma_{2}\right\}$, the chaotic oscillator synchronizes or not to the phase of the dissipative system. The occurrence of this stage produces a resonance regime in the device

linearities which means its resonant and damping terms. Under this condition, a nonlinear controller can control the mechanical system's dynamics if it can match its resonant frequencies. If a feedback signal is sent from the mechanical system to the nonlinear controller then the second can match the phase of the former and enslave its dynamics in a mutual entrainment stage.

Here, two mechanisms are at work corresponding to the phase synchronization of the oscillator's dynamics to the mechanical system's dynamics, and to the resonance induced by the oscillator to the mechanical system (see Fig. 1, cf. Taga 1994). Equation (1) can describe this situation where the system 1 corresponds to the chaotic oscillator and the system 2 to the mechanical system. Here, the coupling parameters $\gamma_{1}$ and $\gamma_{2}$ will "contract" the two systems' dynamics to each other in the sense given by Slotine and Lohmiller (2001) such that, on the one hand, $\gamma_{1}$ will regulate the state of phase synchronization in system 1 with more or less strength by changing the phase $\phi_{1}$ in function of $\phi_{2}$ (same situation as (3) and on the other hand, $\gamma_{2}$ will vary the amplitude of system 1's dynamics and the frequency of system 2's dynamics (its resonance state, cf. (2)).

Under this scheme, synchronization and resonance can rapidly be amplified in few iterations and the systems can then be driven with a very small gain. Fradkov defined this mechanism as feedback resonance (cf. Fradkov 1998, 1999) which permits the discovery and the exploration of one nonlinear system's intrinsic dynamics.

\section{Experiments}

One efficient control gets easier to achieve if the controller incorporates some knowledge about the device to be con- 
trolled, or if the device carries out some parts of the taskwhat is known as computational morphology (Pfeifer et al. 2004) which emphasizes the role of the body's morphology (e.g., its natural synergies and geometrical redundancies) and of its material properties (e.g., the stiffness of the joints) for control. Under these conditions, the partial overlap between the controller and the body's dynamics reduces the complexity of the ensemble. On the one hand, a nonlinear oscillator may achieve so by matching the phase of the device. On the other hand, the morphology and the materials of the mechanical system may ease its action. The rate of PS relates then directly to the areas of good exploitation of the mechanical system (e.g., the motor synergies).

In this part, we investigate the interplay between the controller, the materials and the morphology in three robotic experiments with different architectures and gradual complexity using pneumatic actuators (McKibben type). The first experiment consists on the compliance control of one single artificial muscle. The second experiment considers the torque control of an arm-like mechanical system articulated in the sagittal plane and actuated by two antagonistic air muscles. The third experiment presents a leg-like system with three joint articulations (hip, ankle and knee) aligned agonistically. We provide web links to some supplemental videos of these experiments. ${ }^{1}$

\subsection{Experiments with one Air Muscle}

McKibben actuators are pneumatic rubber tubes confined into a bladder (see Fig. 2a-b). The volume of the bladder is fixed but the pressure inside it and its length can be controlled depending of the amount of air injected into the rubber tube. A small air volume injected will correspond to its resting mode whereas a big air volume injected will correspond to its compressed mode. The pressure $\rho$ in $\mathrm{MPa}$, the nominal length of the bladder $L$ in $\mathrm{m}$, and the diameter $d$ of its surface section $S$ in $\mathrm{m}^{2}$ define their properties such as the springiness or the damping ratio during the nominal and compressed modes (see Klute et al. 1999). We use a muscle with a resting and minimal length of resp. $L=16 \mathrm{~cm} / \mathrm{L}=12 \mathrm{~cm}$ with a diameter $d=1 \mathrm{~cm}$.

In our experiments, we assume that these values are not accessible or defined but have to be retrieved via synchronization. That is, rather than to model the motion coordination, we would like that frequency matching arises from the mutual entanglement between the controller and the air muscle as a by-product of their coupling. Consequently, we choose on purpose as nonlinear controller a discrete chaotic map having fast dynamics but capable to phase-lock its envelop to an external source. We define it with the equation

\footnotetext{
${ }^{1}$ http://www.isi.imi.i.u-tokyo.ac.jp/ alex.
}

McKibben actuator

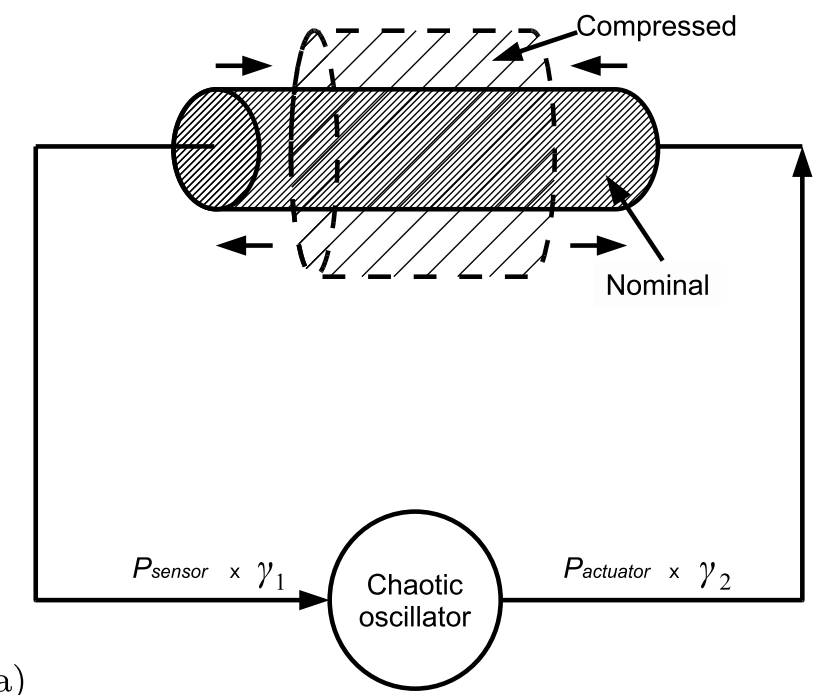

b)

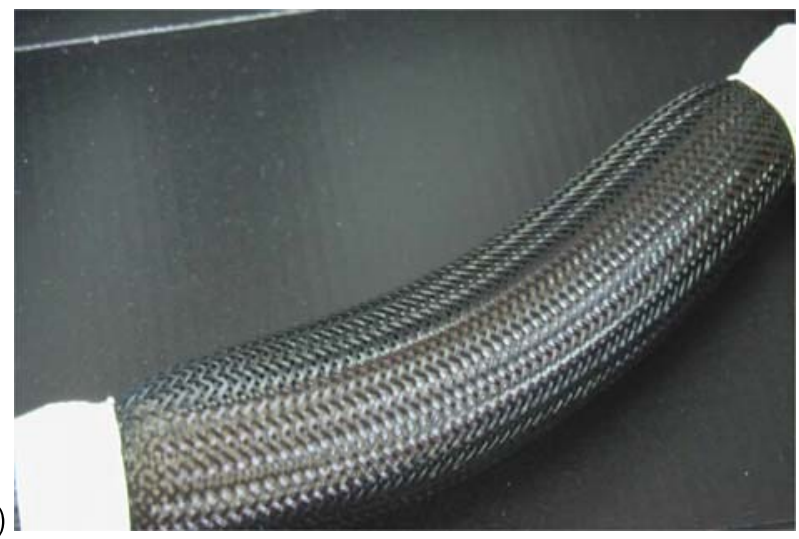

Fig. 2 Controlling loop of the McKibben actuator by a chaotic oscillator. The pair $\left\{\gamma_{1}, \gamma_{2}\right\}$ governs the level of phase synchronization in the chaotic system and the resonant modes in the air muscle. The synchronized oscillator drives the muscle to its resonant frequencies and the stability of the solutions depends on the supplied gain $\gamma_{2}$

of the logistic map $f(z)$ :

$f(z)=1-\alpha z^{2}$,

where the parameter $\alpha$, limited between the interval $[0.0 ; 2.0]$, controls the entropy level of its dynamics which are comprised within the interval $[-1 ; 1]$. We fix also $\alpha$ to 1.95 corresponding to a high level of chaoticity.

The controlling scheme follows a similar setup as the one described in Sect. 2 where the coupling parameters $\left\{\gamma_{1}, \gamma_{2}\right\}$ constrain the dynamics of both systems to each other (see Fig. 2a). On the one hand, the logistic map receives as input the pressure $P_{\text {sensor }}$ of the actuator-measured by a pressure sensor attached at one extremity $(50 \mathrm{~Hz}$ sampling rate) -normalized between $[-1 ; 1]$ and weighted by $\gamma_{1}$. On the other hand, the pneumatic actuator receives the chaotic map's output, $P_{\text {actuator }}$, sampled at $10 \mathrm{~Hz}$ and weighted by 


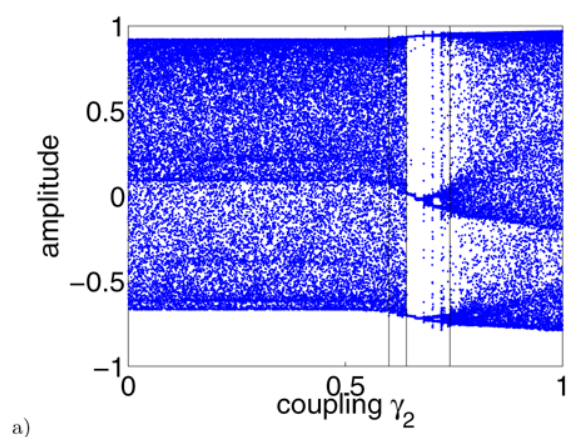

a)
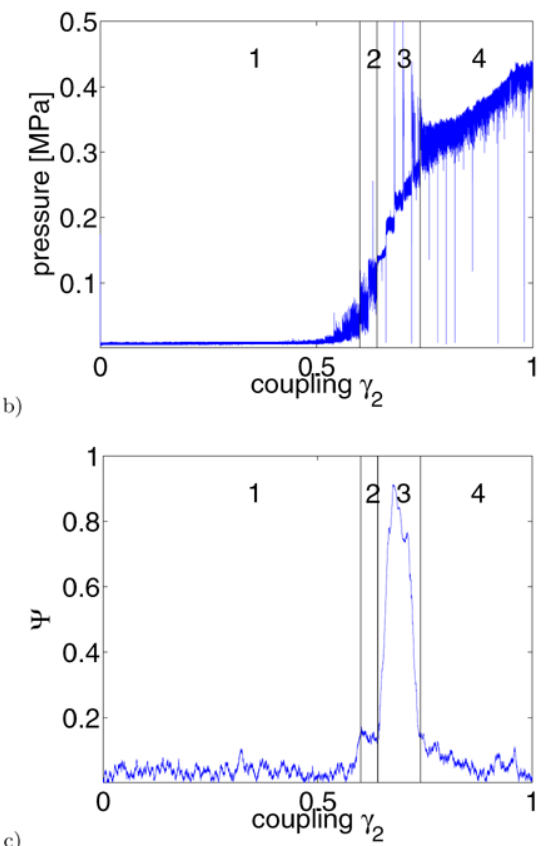

Fig. 3 Controller's amplitude for $\gamma_{2} \in[0,1]$ (a), the corresponding air pressure (in MPa) inside the McKibben actuator (b) and the phase synchronization index $\psi$ between both systems (c). $\psi$ measures the level of phase synchronization between the controller and the air muscle

the coefficient $\gamma_{2}$. The coupling equations are:

$\left\{\begin{array}{l}f(x+1)=\left(1-\gamma_{1}\right) f(x)+\gamma_{1} P_{\text {sensor }}, \\ P_{\text {actuator }}=\gamma_{2} f(x+1)\end{array}\right.$

Here the two terms $\gamma_{1}$ and $\gamma_{2}$ control the functional coupling between the pneumatic actuator and the chaotic oscillator but in order to simplify the analysis we fix one of the parameters, $\gamma_{1}$, to 0.04 .

We study then for which values of $\gamma_{2}$, comprised between $[0 ; 1]$, a phase transition will occur by exploring its interval range. Figures $3 \mathrm{a}-\mathrm{c}$ present the results of the experiment with respectively the amplitude of the chaotic controller, the pressure measured inside the actuator and the relative level of PS calculated with the phase synchronization index ${ }^{2} \psi$. One can observe a clear bifurcation in the controller's dynamics for a specific coupling value $\gamma_{2}$, its modulation creates therefore the conditions for various behaviors in the McKibben actuator.

We distinguish three different cases for different $\gamma_{2}$ intervals and four regions annotated in the figures:

- Case 1. For $\gamma_{2}<0.60$, PS does not occur $(\psi<0.10)$ and the McKibben actuator is fully passive. The supplied pressure in the air muscle is too weak and noisy to generate any variability, it remains inferior to $0.01 \mathrm{MPa}$. This situation corresponds to area 1 in Fig. 3 where the chaotic map stands for a noisy energy supplier to the pneumatic muscle. Their respective dynamics are displayed in Fig. 4a.

- Case 2. For $\gamma_{2} \in[0.60 ; 0.74]$, a bifurcation occurs inside the systems' dynamics. In this new stage, the chaotic map synchronizes its phase to the intrinsic frequency of the McKibben actuator which is entrained into resonance. Here, the controller is settled into three states (i.e., three synergies): $f(x)=-0.75$ which corresponds to the resting mode, $f(x)=+1.0$ which corresponds to the compressed mode, and $f(x)=0.0$ which is a mode in-between that attempts to combine both of them. Like for the real muscles, the McKibben actuator can be activated by concurrent motor commands and tries to integrate them into a novel mode (cf. Bizzi and Clarac 1999). The actuator is in a sort of multistable state.

We can distinguish two regions that constitute the PS area, resp. region 2 for $\gamma_{2} \in[0.60 ; 0.66]$ and region 3 for $\gamma_{2} \in[0.66 ; 0.74]$. Region 2 corresponds to a situation of weak synchronization between the two systems $(\psi \approx 0.18)$. In that stage, the rubber tube is transiently entrained into resonance but not stabilized. This produces a high variability inside the tube and nonlinear stiffness; the compliance inside the air muscle changes rapidly by elongating and contracting the actuator's length dynamically. Since the material is very sensitive to the signal phase, small variations of $\gamma_{2}$ in this interval range produce important qualitative changes inside the rubber tube.

For $\gamma_{2} \in[0.66 ; 0.74]$ in region 3 , the global system reaches its nominal regime. The rubber tube has a stable pressure rate of $0.25 \mathrm{MPa}$ with stable periodicity; see Fig. $3 \mathrm{~b}$ and Fig. $4 \mathrm{~b}$. The two systems are in phase and $\psi$ culminates to its highest level (see Fig. 3c). Compared to region 2 , we emphasize that the regime developed in

${ }^{2}$ The phase synchronization index $\psi$ between two signals $A$ and $B$ is defined as

$\psi=\sqrt{\langle\cos \varphi(t)\rangle^{2}+\langle\sin \varphi(t)\rangle^{2}}$

with \langle\rangle the mean function and $\varphi(t)=\phi_{A}(t)-\phi_{B}(t)$ the phase difference of the two signals. $\psi$ normalizes the phase synchronization rate within the interval $[0,1]$ (cf. Pikovsky et al. 2001). 
Fig. 4 Dynamical transition of the valve behavior for different $\gamma_{2}$. For $\gamma_{2}=0.50$, no synchronization occurs in the chaotic map and the pressure in the actuator is low (a). For slight variations of $\gamma_{2}$ value above 0.60 , the chaotic map synchronizes to the resonant frequencies of the pneumatic muscle (at $3.33 \mathrm{~Hz}$ ) exhibiting that the McKibben actuator is sensitive to the controller phase (b-c); resp. for $\gamma_{2}=0.66$ and 0.76 . Hence, dynamical synchronization depends on the coupling term $\gamma_{2}$ and a too high gain destroys the phase-locking to the McKibben dynamics and saturates its dynamics (d); resp. $\gamma_{2}=0.90$ a)
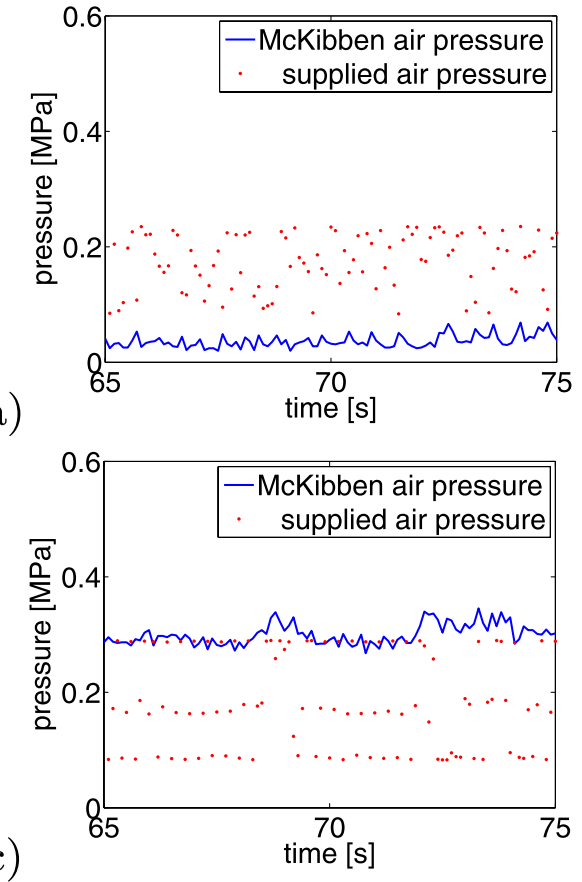

b)
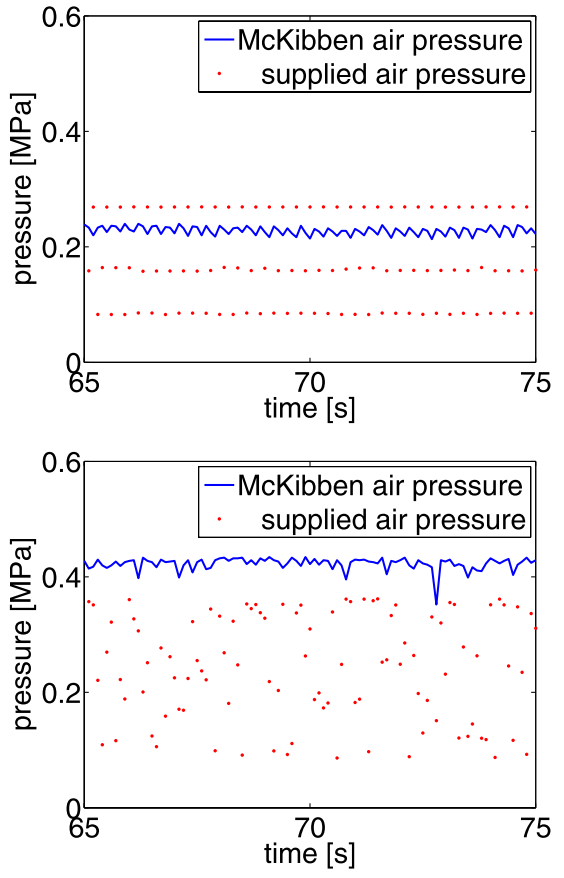

the rubber tube here is also sensitive to the phase of the control signal and not to its amplitude because $\gamma_{2}$ varies only slightly. It corresponds to the state of PS $(\psi \approx 0.8)$ when the chaotic map synchronizes to the resonant frequencies of the McKibben actuator and entrains it to its stable rhythm and nominal pressure.

- Case 3. For $\gamma_{2}>0.74$ (region 4), the high gain imposed to the pneumatic muscle saturates its dynamics. As a result, the synchronous regime of region 3 does not pertain. The chaotic map follows simultaneously three attractors that overlap more and more as $\gamma_{2}$ augments; the rhythm vanishes and $\psi$ returns to a low value. The air muscle becomes stiffer and less dynamic (see Fig. 4c-d resp. for $\gamma_{2}=0.76$ just above the interval of PS and $\gamma_{2}=0.90 \mathrm{far}$ from it).

To resume, $\gamma_{2}$ controls dynamically the coupling strength between the chaotic map and the artificial muscle, and modulates their phase relations. We analyze further this situation in the temporal and spectral domains with the phase plot of the McKibben actuator dynamics displayed in Fig. 5 for the three different cases and the spectral bifurcation diagram (SBD, ${ }^{3}$ Orrel and Smith 2003) of the two systems respectively in Fig. 6a-b. First, the phase plot in Fig. 5 presents the

\footnotetext{
${ }^{3}$ In order to investigate qualitative changes of the dynamics in the spectral space we use the spectral bifurcation diagram (SBD, cf. Orrel and Smith 2003). Essentially, SBD displays the power density spectrum of multiple system variables as a function of a system control parameter (e.g., force, temperature, coupling strength). This method allows identification of the resonant states characterized by sharp frequency components, chaotic states having rather broad power spectra, as well as bifurcation, that is, qualitative changes in the system's behavior (phase
}

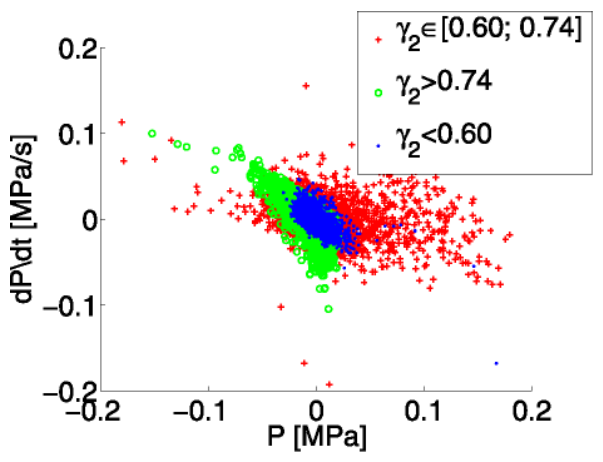

Fig. 5 (Color online) Phase plot of the air pressure inside the McKibben actuator. Mapping of the three different cases corresponding to the elongation mode $\gamma_{2}<0.60$ (blue dots); to the compliant mode (PS) $\gamma_{2} \in[0.60 ; 0.74]$ (red crosses) and to the compressed mode $\gamma_{2}>0.74$ (green circles)

air pressure flow relative to its first derivative (zero-centered for all the cases) for the three cases: $\gamma_{2}<0.60$ when the two systems interact weakly from each other (blue dots), $\gamma_{2} \in[0.60 ; 0.74]$ of mutual entrainment, at PS, when the controller exploits optimally the dissipative system and develops high variability (red crosses), and $\gamma_{2}>0.74$ when a strong air pressure saturates the muscle (green circles). Second, the spectral bifurcation diagram plots the power spectrum densities of the chaotic controller and of the air muscle relative to the parameter $\gamma_{2}$, see Fig. $6 \mathrm{a}-\mathrm{b}$. It exhibits the re-

transitions from one state attractor to another). The spectral bifurcation is the Fourier transform performed for different values of the control parameter. 
chaotic map's dynamics

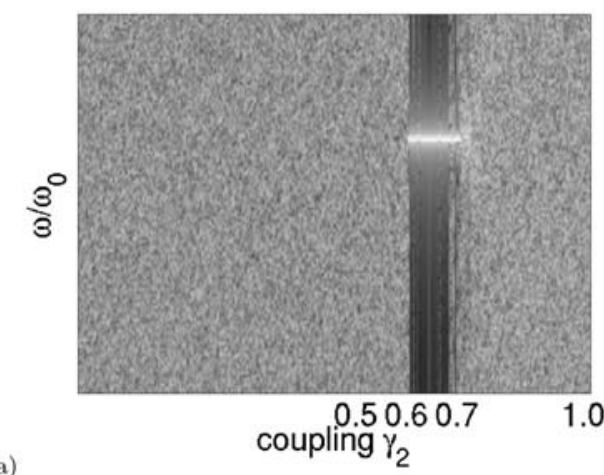

a)

Fig. 6 Spectral bifurcation diagram of the chaotic map dynamics (a) and of the McKibben actuator internal pressure (b). It reveals the region of mutual entrainment (i.e., resonant frequencies) relative to the control parameter $\gamma_{2}$ between $[0.0 ; 1.0]$. For $\gamma_{2}<0.60$, the two systems

gion where the chaotic map matches the resonant frequency of the air muscle at PS at $3.33 \mathrm{~Hz}$.

\subsection{Joint control of 1 DOF arm with antagonistic muscles}

The previous experiment described how synchronization creates a control interface at the material level that can modulate the stiffness and the compliance of one muscle. In this Section, we extend its action to the morphological level via the physical constraints of a body structure. We employ to this end a elbow-like mechanical system actuated by two air muscles (see Fig. 7). The system is composed of two linked bars (1 DOF joint rotation) where the lower-part is fixed at the base-ground and the upper-part moves freely in the sagittal plane.

The two actuators are disposed antagonistically such that they control concurrently the angular position $\theta$ between the two bars (measured by an angular position sensor). This structural constraint eases the contiguous elongations and contractions to act either in opposition or in unison. The system has therefore some phase dependencies relative to the material properties and others relative to the body's morphology (its geometry) that a motor synergy should incorporate. In our synchronization scheme, this state will occur if the controller adapts its phase to the muscles' characteristics (their resonant frequencies) and to the body's architecture for specific coupling.

Here, each chaotic map controls exclusively one muscle like in Sect. 3.1 using the same equations; i.e., (7). The two controllers do not have any explicit coupling to each other but are rather externally linked through the body. Since we assume that the same pair $\left\{\gamma_{1}, \gamma_{2}\right\}$ controls both actuators, the pair operates therefore a dimensionality reduction of the control task. In the following, we explore the interval ranges that exhibit the different synchronization regimes possible to
McKibben's internal air pressure

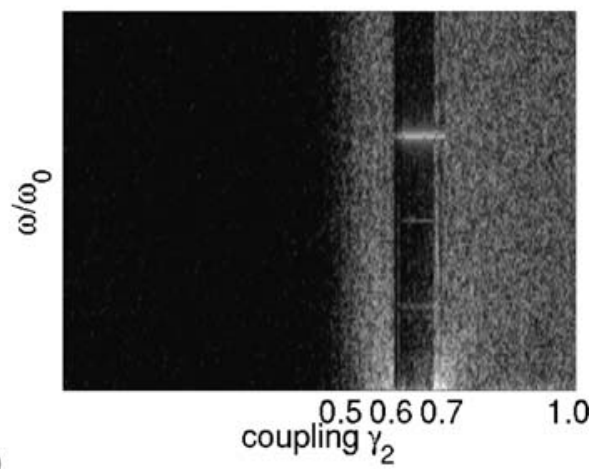

b)

interact loosely with no definite bandwidth whereas for $\gamma_{2}>0.74$, the too high gain saturates the dynamics. In contrast, for $\gamma_{2} \in[0.60 ; 0.74]$, the chaotic map synchronizes its phase to the fundamental frequency of the pneumatic muscle at $3.33 \mathrm{~Hz}$

achieve and we analyze the conditions that enable the control of the elbow-like articulation.

For this, we fix $\gamma_{2}=0.60-$ where synchronization occurred in the previous experiment-and set $\gamma_{1}$ to three different values corresponding to three different states observed; see, Fig. 8: (a) without external feedback $\left\{\gamma_{1}, \gamma_{2}\right\}=$ $\{0.00,0.60\}$, (b) under a weak external influence $\left\{\gamma_{1}, \gamma_{2}\right\}=$ $\{0.04,0.60\}$, (c) under a strong external influence $\left\{\gamma_{1}, \gamma_{2}\right\}=$ $\{0.18,0.60\}$. The diagrams on the left side reproduce the time series of the two controllers whereas those on the right side reproduce the time series of the joint angle $\theta$. Figure 9 displays their respective phase plots.

For $\gamma_{1}=0.00$ (without feedback), the oscillators are open-loop and because their dynamics are not constrained by any external loads, they develop a very noisy output. Meanwhile, they do not entrain the limbs and the joint angle remains fixed $(\theta=1.3 \mathrm{rad})$; the system is passive and released. In contrast, when the controllers receive a weak input $\left(\gamma_{1}=0.04\right)$, a phase transition occurs at the body level, see Fig. 8b: after a transient period of high variability and disorganized dynamics, the system stabilizes itself into a limit cycle where the two oscillators start to work in phase, see Figs. $8 \mathrm{~b}$ and 9 . Since the amplitude of the chaotic maps' output remains fixed due to $\gamma_{2}$, the difference with the previous case $\gamma_{1}=0.00$ comes from a change in the temporal domain (phase). Here, the controllers' dynamics exploit the phasic information of the body to concurrently discover its nominal regime (i.e., intrinsic resonant forces) and the joint angle develops a maximal angular amplitude when the individual controllers lock to its phase. At reverse, if a too high input gain is applied, which corresponds to $\gamma_{1}=0.18$, the controllers are saturating their dynamics and the mechanical system develops a very strong contraction.

PS acts therefore both at the material and morphological level to generate different dynamics such as the arm's os- 


\subsection{Joint control of a 3 DOF leg with ground contact}

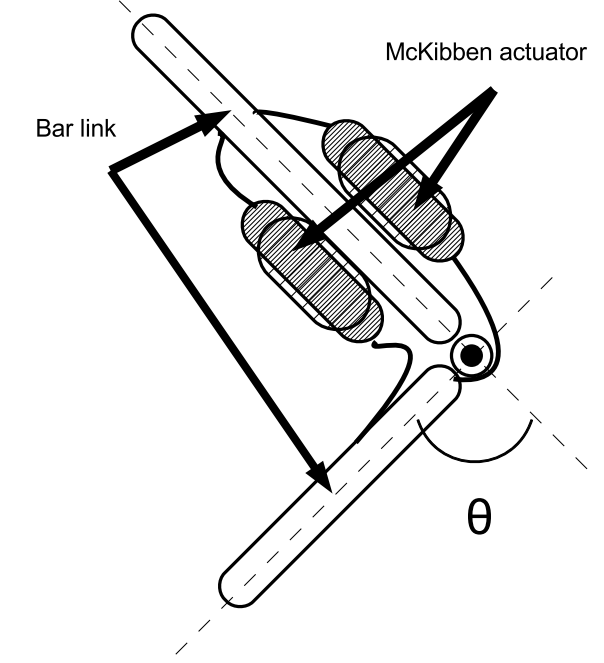

a)

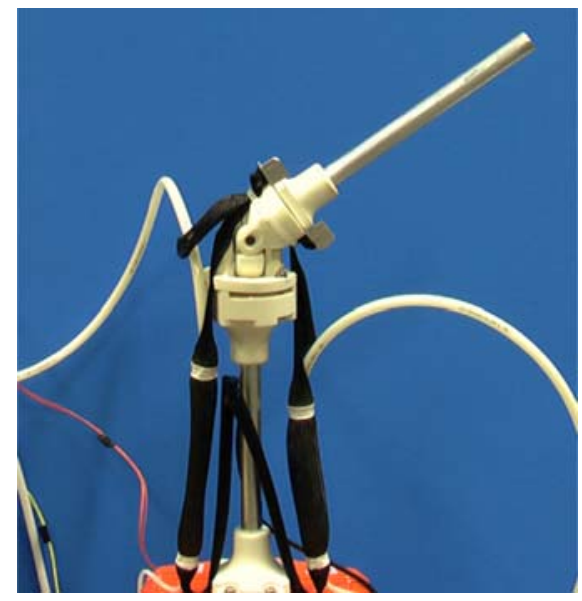

b)

Fig. 7 Articulated joint (1 DOF) actuated by two antagonistically positioned air muscles. The air pressure inside the two limbs are regulated with two different control commands (chaotic oscillators). Stable oscillatory movements are produced if we have a phase coordination in both (1) the internal and the body-environment dynamics and (2) between the two controllers

cillation, its strong contraction or its release. Similar to the top-down chemical neuro-transmittors that change the functional configuration of CPGs to produce different motor outputs, the pair $\left\{\gamma_{1}, \gamma_{2}\right\}$ modulates the controllers' dynamics to achieve different types of behavior. Therefore, they act more as a filter for processing sensory information rather than a generator of commands (Kuo 2002), and the body's morphology contributes to the control (Pfeifer et al. 2007; McMillen et al. 2008).
The materials and the body structure have a strong influence on the type of functions and behaviors possible to develop; nevertheless, the physical interaction with the environment is also essential like during walking or hopping (Lungarella and Berthouze 2002; Seyfarth et al. 2007; Rummel et al. 2008). We use a leg-like system to explore this issue. Three joint angles constitute its mechanical architecture which replicate the ankle-knee-hip articulation of vertebrates' legs (see Fig. 10). The three segments constitute the foot, the tibia and the femur and measure each $18 \mathrm{~cm}$. Moreover, two artificial air muscles actuate the ankle and the knee joints respectively whereas the hip joint (the upperpart) is passive. Here, the neural impulses have to incorporate some knowledge about the geometry of the limbs and the material properties in order to produce some loose control. We want to explore to what extend transient synchronization and entrainment deal with load changes during the swing period and the stance period.

To this purpose, we use in this experiment a more realistic biological neuron model that replicates the spiking frequency adaptation of the neurons in the spinal cord. Its dynamics are defined by the formal model proposed by Izhikevich (2004) which approximates the Hodgin-Huxley model. Its equations are:

$$
\left\{\begin{array}{l}
v^{\prime}=0.04 v^{2}+5 v+140-u+I \\
u^{\prime}=a(b v-u)
\end{array}\right.
$$

with $v$ representing the membrane potential of the neuron in $\mathrm{mV}$ and $u$ a membrane recovery variable, $v^{\prime}$ and $u^{\prime}$ are their respective temporal derivatives.The signal $I$ triggers the neuron's membrane potential and can lead to a spike if it crosses the voltage threshold of $30 \mathrm{mV}$. The after-spiking rule is:

if $v \geq+30 \mathrm{mV}, \quad$ then $\left\{\begin{array}{l}v \leftarrow c \\ u \leftarrow u+d,\end{array}\right.$

where the variables set $\{a, b, c, d\}$ defines the neuron's attributes: the values $(a ; b)=(0.02 ;-0.1)$ and $(c ; d)=$ $(-55 ; 6)$ determine a class 1 excitable neuron whose spiking frequency is proportional to the trigger amplitude $I$. In our experiments, we limit I's values between $24 \mathrm{~mA}$ (corresponding to low frequency adaptation) to $100 \mathrm{~mA}$ (corresponding to high frequency adaptation). We also threshold the neuron voltage $v$ so that the volume of air sent $P_{\text {actuator }}$ is maximal when $v>-60 \mathrm{mV}$ and minimal when $v<$ $-60 \mathrm{mV}$. This operation transforms a spike train (e.g., three spikes) into one step function of equal interval length which permits to relieve the air compressors' work while preserving the phase information. The gain $v$ is then weighted by the coupling parameter $\gamma_{2}\left(\gamma_{2}=1.00\right)$. 
Fig. 8 Dynamics of the two air muscles dynamics (left column) and of the associated joint angle (right column) for the three cases (a) no input

$\left\{\gamma_{1}, \gamma_{2}\right\}=\{0.00,0.60\}$,

(b) weak input

$\left\{\gamma_{1}, \gamma_{2}\right\}=\{0.04,0.60\}$,

(c) strong input

$\left\{\gamma_{1}, \gamma_{2}\right\}=\{0.18,0.60\}$.

Maximal angular amplitude is developed when the individual controllers work in phase with a rhythm that depends on their body locations, case (b) a)
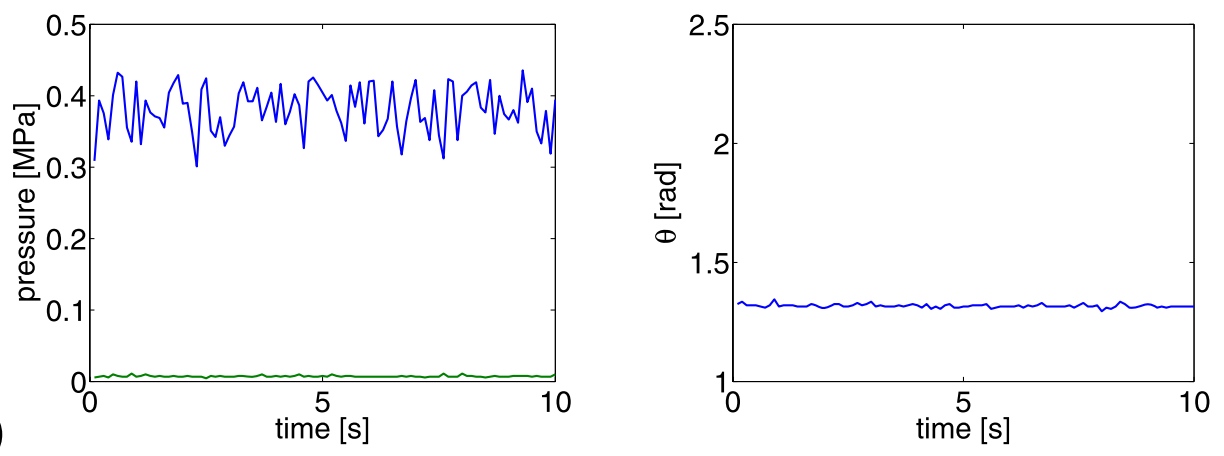

b)
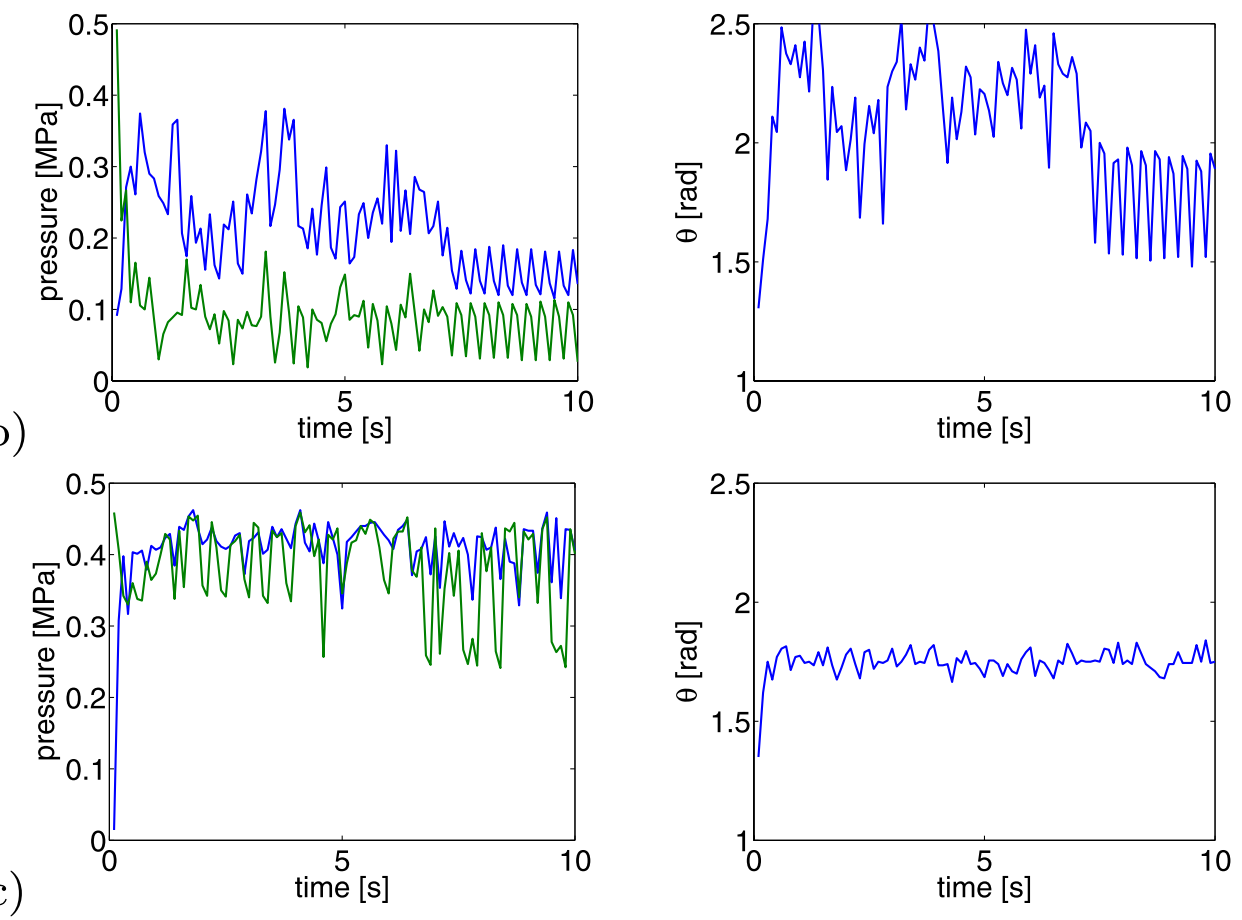

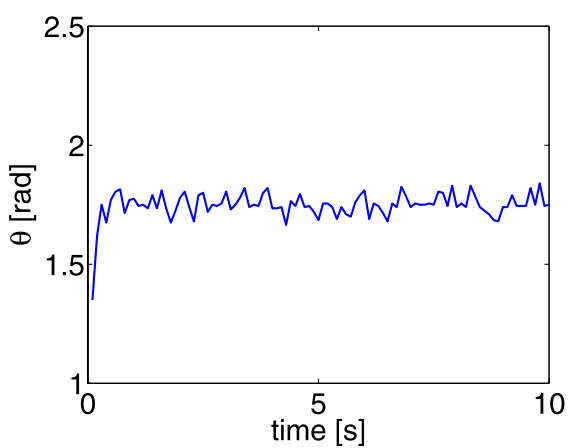

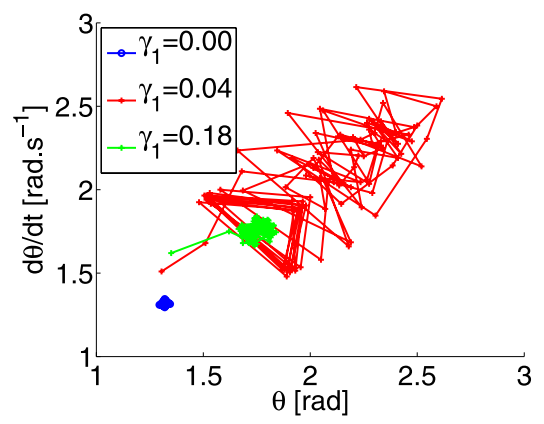

Fig. 9 Phase plots of the 1-DOF joint angle corresponding to the three cases of Fig. 8: $\gamma_{1}=0.00$ (no sensory feedback), 0.04 (weak coupling), 0.18 (strong coupling) for fixed output gain $\gamma_{2}=0.60$ (cf. Fig. 8). The fed back information from the sensors has a structuring effect on the dynamics of the oscillators. For weak coupling, $\gamma_{1}=0.04$, the result is the motion's stabilization of the system to an oscillatory regime; the oscillators' phase synchronize to the body intrinsic frequency

We measure the air volume inside each muscle $P_{\text {sensor }}$ using pressure sensors attached at one extremity. Each neuron receives then the current input $I=I_{0}+\gamma_{1} P_{\text {sensor }}\left(I_{\text {lim }}-I_{0}\right)$, where $I_{0}$ is the static current, $\gamma_{1}$ the control parameter that we vary within the interval $[0 ; 1] ; I_{0}=24 \mathrm{~mA}$ and $I_{\lim }=$ $100 \mathrm{~mA}$. The frequency of the spikes depends therefore on the amplitude of the current injected but also on the physical constraints induced in the air muscle during ground contact. We speculate that for specific coupling and body posture, the two controllers are able to mutually entrain their dynamics to a certain phase delay corresponding to the particular muscles disposition of the leg. To this end, we propose to analyze the system's behavior when we vary the parameter $\gamma_{1}$ to different values. We plot in Fig. 11a-c the respective time series of the two spiking neurons (left column), and the respective knee and ankle vertical displacement in relative coordinates retrieved from motion capture (right column) for the three cases $\gamma_{1}=0.11, \gamma_{1}=0.17$, and $\gamma_{1}=0.25$. We reproduce in Fig. 12 the displacements of the knee, ankle and hip in absolute coordinates in the sagittal plane for these three situations. 


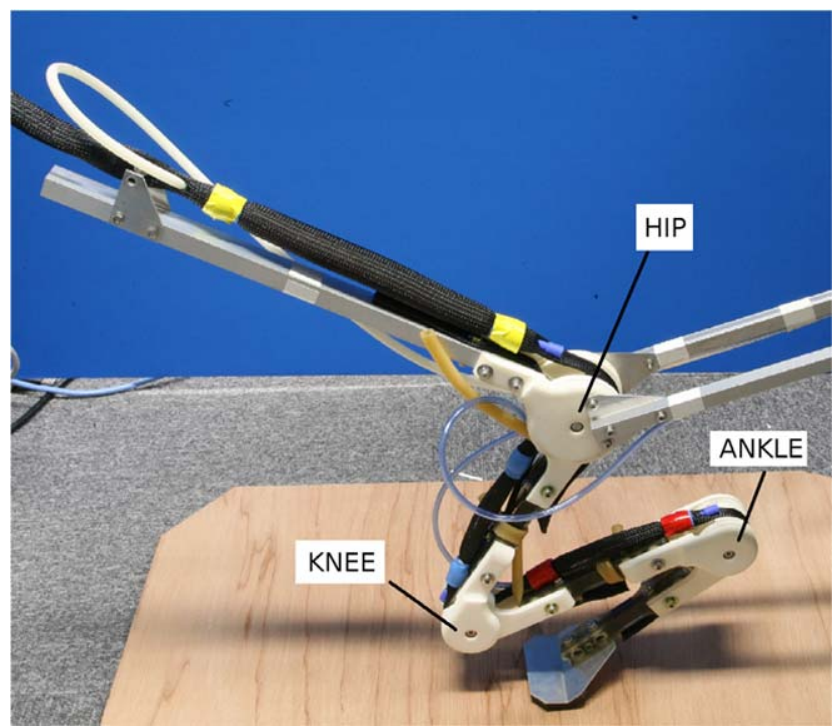

Fig. 10 Leg-like articulation

For $\gamma_{1}=0.11$ in Fig. 11a, the leg executes very little hops which correspond mostly to reflexive patterns (blue dots in Fig. 12). The oscillators are not completely in phase due to their weak coupling and generate spikes of $13 \mathrm{~ms}$ of interval range. They perturb the body's passive dynamics such that the joint angles absorb the impulse with a damped oscillation followed by a rebound; see Fig. 11a in the right column.

The situation however changes for $\gamma_{1}=0.17$ in Fig. 11b where the system develops its optimal regime and generates rhythmic jumps of higher amplitude. Here, the oscillators are in phase and the ankle and the knee develop their maximal amplitude of motion. The gait combines one harmonic frequency to the fundamental frequency in a bimodal rhythm. The oscillators actively entrain the leg and exploit its dynamic. This occurs when the spike trains are more numerous and their interval range longer between 15 and $20 \mathrm{~ms}$. Although the coordination is much more flexible, the behavioral pattern is rather stable (the red circles in Fig. 12).

For greater values like $\gamma_{1}=0.25$, this interval of good coupling in the parameter space however vanishes see Fig. 11c. The stronger the input current is, the more the oscillators generate spikes that influence in return the muscle's activation phase length (approx. $30 \mathrm{~ms}$ ). As a result, the motion behavior, which is apparent to a forced regime, embeds more harmonics that disrupt a little more the coordination, see the green crosses in Fig. 12.

\section{Conclusion}

In this paper, we presented a standard control scheme based on the phenomenon of phase synchronization that mimics the action of neuromodulators to adjust the neural con- trollers to the natural dynamics of a mechanical system. In biological systems, neuromodulators permit the fine tuning of the central pattern generators to specific dynamics which include not only their proper rhythms but also those imposed by the body itself (visco-elastic properties, morphology) and those imposed by the higher decision commands in the central nervous system. They contribute therefore to a decentralized view of control that may ease the discrimination of the regions of interest in the parameters space, reduce the complexity of control and enhance energy efficiency.

Since the visco-elastic properties of the materials and the body morphology play some roles in the control, they affect therefore the size of the region of interest (of useful control) in the parameters space: e.g., for the syncing of one air muscle, the rhythmic and angular control of the arm-like joint and the entrainment of the leg-like articulation. For instance, $10 \%$ of the space only in Fig. 3 (regions 2-3) leads to the oscillatory regime, this surface depends on the visco-elastic properties of the material: the more compliant the material is, the larger the interval. However, global entrainment cannot withstand all non-stationary situations and entrainment will fail under some given load situation. A higher controller may advantageously switch between different synergies to modify the stiffness of the muscles at precise periods only so that the most efficient synergy is selected for sudden disturbances: e.g., stiff muscles during the stance phase only (region 4 in Fig. 3) then more compliant muscle during the swing phase (regions $2-3$ in Fig. 3). Ting and colleagues (Ting and MacPherson 2005; Ting 2007) support such scenario-which updates the motor synergy concept defined by Bizzi and Clarac (1999) and propose that the synergies can be selected or dismissed "on demand" from a set of synergies for specific postural tasks. In line with them Ivanenko et al. (2005) propose that voluntary motor programs of higher-level control signals are just simply "inserted" into the locomotion program at a precise timing. Therefore, a hierarchical view of movement control with on the one hand a repertoire of motor synergies executed by neurons (alpha-motoneurons) controlled by few coupling parameters (neuromodulators) and on the other hand a higher system that exploits efficiently the first structure may ease the controllability problem and reduce its dimensionality. This follows one of the idea presented by Latash et al. (2007) on motor synergies and of contraction theory (Lohmiller and Slotine 1998; Slotine and Lohmiller 2001; Seo and Slotine 2007). Although more oscillators to entrain imply more combinations, the body morphology of vertebrates possesses lots of regularities that greatly restrict the number of pertinent coordinations (e.g., symmetries of the legs and the arms dynamics during walking). A learning algorithm could thus discover the useful regions of the parameters space in a kind of trial and error exploration, then exploit and combine them to generate fast muscles grouping or postural control when needed. 
Fig. 11 Time series of the two spiking neurons (left column) and the relative knee and ankle vertical displacements (right column) for the three cases $\gamma_{1}=0.11, \gamma_{1}=0.17$, and $\gamma_{1}=0.25$, resp. (a), (b) and (c). Small coupling values $\left(\gamma_{1}=0.11\right)$ perturb only the body's passive dynamics whereas stronger ones $\left(\gamma_{1}=0.25\right)$ destroy coordination. In between for $\gamma_{1}=0.17$, the oscillators actively entrain the body dynamics to resonance

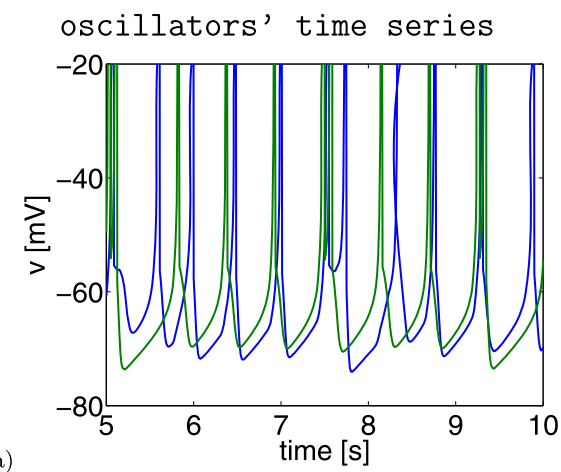

knee \& ankle vertical displacements

a)
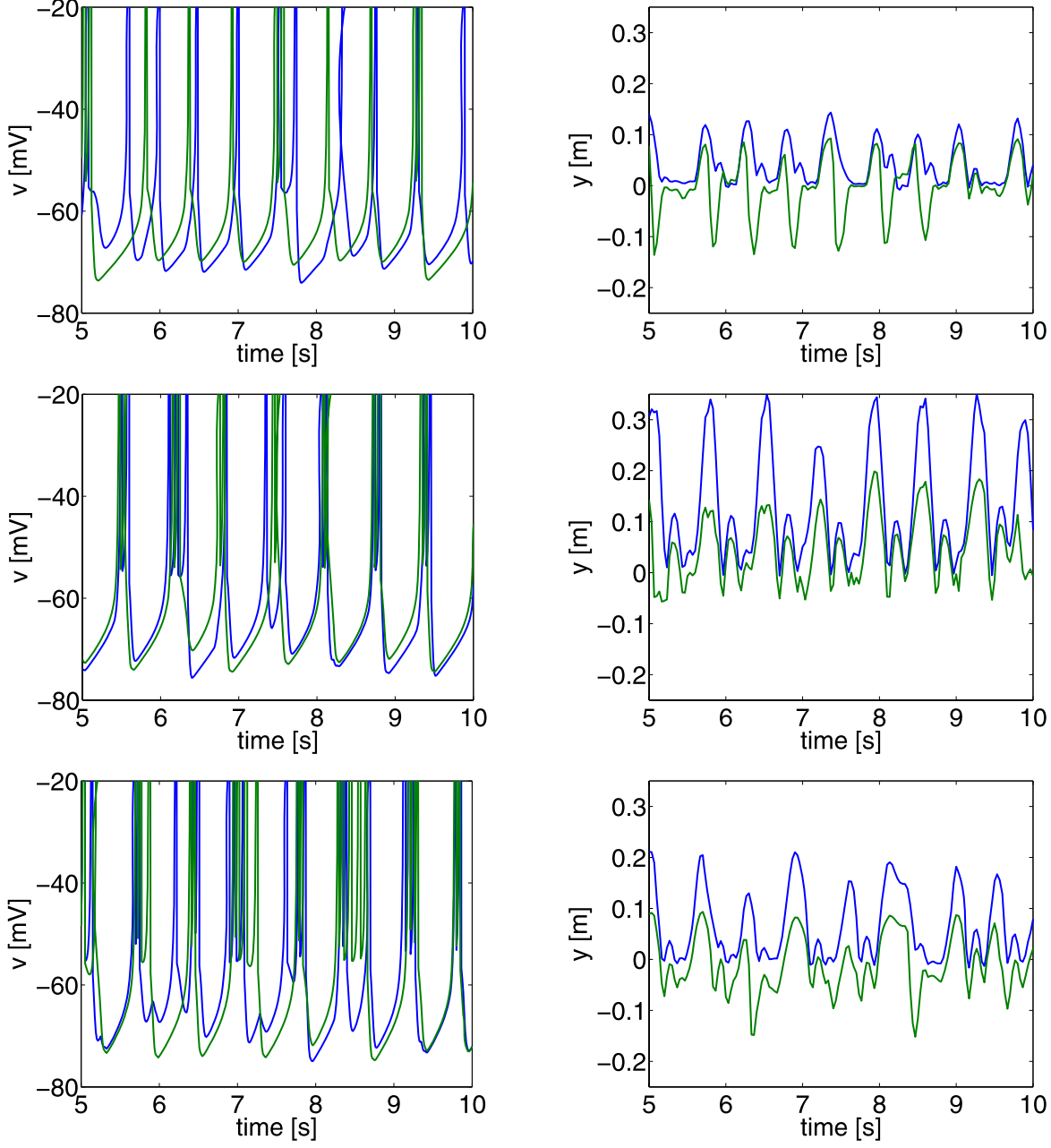

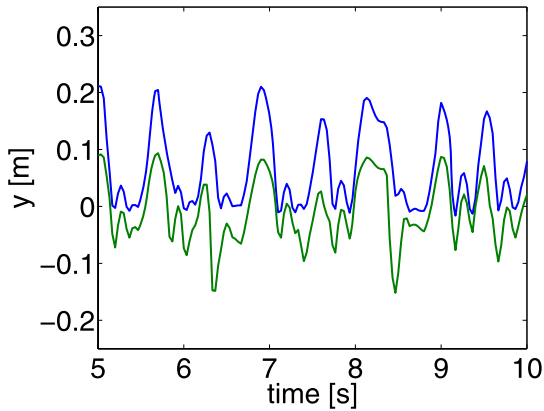

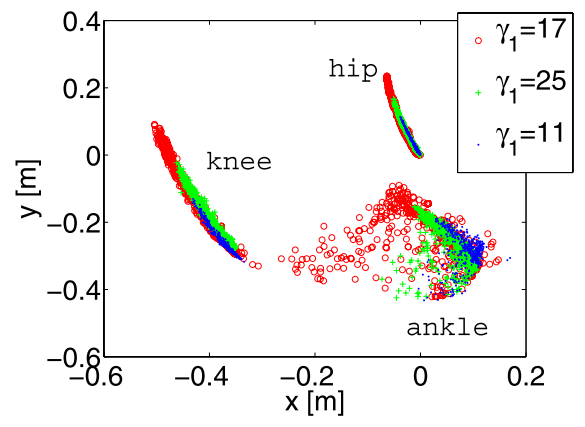

Fig. 12 (Color online) Diagram of the horizontal and vertical displacements of the hip, knee and ankle in the sagittal plane for three cases described in Fig. 11. The blue dots present the situation for $\gamma_{1}=0.11$, the red circles present the situation for $\gamma_{1}=0.17$, and the green crosses present the situation for $\gamma_{1}=0.25$

A similar scenario is advocated to occur during infant's development to shape the formation of spinal organization and cortical maps (cf., Schouenborg 2003; Petersson et al. 2004; Granmo et al. 2008).
Phase synchronization has been already considered in other works for tuning in the internal controllers to various regimes of coordination with the body dynamics. One difference with them in this work is perhaps that the rhythmical gait patterns are not present in the internal controllers but created dynamically by exploiting the body. At least in the first two experiences, the chaotic maps do not have any clear limit cycles, the interacting systems create dynamically the rhythmical pattern. In many models of CPGs, the oscillators have an explicit frequency defined parametrically or learned such as the Kuramoto oscillator, the Rossler oscillator or any sinusoidal functions. However, experiment 3 is a little more in line with these frameworks since we modulate purposefully the internal frequency of each oscillator. There, the oscillators entrain the body dynamics with appropriate phase lags.

Acknowledgements The authors would like to thank the reviewers for their useful comments and to acknowledge the Asada ERATO Synergistic project which provided the grant for this research. 


\section{References}

Bizzi, E., \& Clarac, F. (1999). Motor systems. Current Opinion in Neurobiology, 9(6), 659-662.

Bizzi, E., Giszter, S., Loeb, E., Mussa-Ivaldi, F., \& Saltiel, P. (1995). Modular organization of motor behavior in the frog's spinal cord. Trends in Neurosciences, 18, 442-445.

Buchli, J., Righetti, L., \& Ijspeert, A. (2006). Engineering entrainment and adaptation in limit cycle systems - from biological inspiration to applications in robotics. Biological Cybernetics, 95(6), 645664.

Calabrese, R. (1995). Oscillation in motor pattern-generating networks. Current Opinion in Neurobiology, 5, 816-823.

Der, R., Hesse, F., \& Martius, G. (2006). Rocking stamper and jumping snake from a dynamical system approach to artificial life. Adaptive Behaviors, 14, 105-115.

Fellous, J., \& Linster, C. (1998). Computational models of neuromodulation. Robotics and Autonomous Systems, 10, 771-805.

Fradkov, A. L. (1998). Exploring nonlinearity by feedback. Memorandum 1447, Faculty of Mathematical Sciences, University of Twente, The Netherlands, June 1998.

Fradkov, A. L. (1999). Investigation of physical systems by means of feedback. Automation and Remote Control, 60(3).

Gonzalez-Miranda, J. (2004). Synchronization and control of chaos, an introduction for scientists and engineers. London: Imperial College Press.

Granmo, M., Petersson, P., \& Schouenborg, J. (2008). Action-based body maps in the spinal cord emerge from a transitory floating organization. The Journal of Neuroscience, 28(21), 5494-5503.

Grillner, S. (1996). Neural networks for vertebrate locomotion. Scientific American, 274, 64-69.

Grillner, S. (2006). Biological pattern generation: the cellular and computational logic of networks in motion. Neuron, 52, 751-766.

Hosoda, K., Takuma, T., Nakamoto, A., \& Hayashi, S. (2008). Biped robot design powered by antagonistic pneumatic actuators for multi-modal locomotion. Robotics and Autonomous Systems, 56(1), 46-53.

Iida, F., \& Pfeifer, R. (2004). Self-stabilization and behavioral diversity of embodied adaptive locomotion. In F. Iida et al. (Eds.), Embodied artificial intelligence. LNCS/AI (vol. 3139, pp. 119-128). Berlin: Springer.

Ijspeert, A., Nakanishi, J., \& Schaal, S. (2003). Learning attractor landscapes for learning motor primitives. Advances in Neural Information Processing Systems, 15, 1547-1554.

Ijspeert, A. J. (2008). Central pattern generators for locomotion control in animals and robots: a review. Neural Networks, 21(4), 642653.

Ivanenko, Y., Cappellini, G., Dominici, N., Poppele, R., \& Lacquaniti, F. (2005). Coordination of locomotion with voluntary movements. Journal of Neuroscience, 25(31), 7238-7253.

Izhikevich, E. (2004). Which model to use for cortical spiking neurons? IEEE Transactions on Neural Networks, 15(5), 1063-1070.

Kelso, J. (1995). Dynamic patterns: the self-organization of brain and behavior. Cambridge: MIT Press.

Kelso, J. A. S., \& Haken, H. (1995). New laws to be expected in the organism: synergetics of brain and behavior. In M. Murphy \& L. O'Neil (Eds.), What is life? The next 50 years (pp. 137-160). Cambridge: Cambridge University Press.

Klute, J., Czerniecki, G., \& Hannaford, B. (1999). McKibben artificial muscles: pneumatic actuators with biomechanical intelligence. In International conference on advance intelligent mechatronics.

Kuniyoshi, Y., \& Sangawa, S. (2006). Early motor development from partially ordered neural-body dynamics - experiments with a cortico-spinal-musculo-skeletal model. Biological Cybernetics, 95(6), 589-605.
Kuniyoshi, Y., \& Suzuki, S. (2004). Dynamic emergence and adaptation of behavior through embodiement as coupled chaotic field. In Proceedings of 17th international conference on intelligent robots and systems (pp. 2042-2048).

Kuo, A. (2002). The relative roles of feedforward and feedback in the control of rhythmic movements. Motor Control, 4, 129-145.

Latash, M., Scholz, J., \& Schöner, G. (2007). Toward a new theory of motor synergies. Motor Control, 11, 276-308.

Levi, R., Varona, P., Arshavsky, Y., Rabinovich, M., \& Selverston, A. (2005). The role of sensory network dynamics in generating a motor program. The Journal of Neuroscience, 25(42), 9807-9815.

Lockhart, D., \& Ting, L. (2007). Optimal sensorimotor transformations for balance. Nature Neuroscience, 10, 1329-1336.

Lohmiller, W., \& Slotine, J. J. E. (1998). On contraction analysis for nonlinear systems. Automatica 34(6).

Lungarella, M., \& Berthouze, L. (2002). On the interplay between morphological, neural, and environmental dynamics: a robotic case study. Adaptive Behavior, 10(3/4), 223-241.

Lungarella, M., \& Berthouze, L. (2004). Robot bouncing: on the synergy between neural and body dynamics. In F. Iida, R. Pfeifer, L. Steels, \& Y. Kuniyoshi (Eds.), Embodied artificial intelligence. LNCS/AI (vol. 3139, pp. 87-97). Berlin: Springer.

Lungarella, M., Pitti, A., \& Kuniyoshi, Y. (2007). Information transfer at multiple scales. Physical Review E, 76(5), 056117, part 2.

Marder, E. (1996). Neural modulation: following your own rhythm. Current Biology, 6(2), 119-121.

Marder, E., \& Calabrese, R. (1996). Principles of rhythmic motor pattern production. Physiological Reviews, 76, 687-717.

McMillen, T., Williams, T., \& Holmes, P. (2008). Nonlinear muscles, passive viscoelasticity and body taper conspire to create neuromechanical phase lags in anguilliform swimmers. PLoS Computational Biology, 4(8), e1000, 157.

Nakanishi, J., Morimoto, J., Endo, G., Cheng, G., Schaal, S., \& Kawato, M. (2004). An empirical exploration of phase resetting for robust biped locomotion with dynamical movement primitives. In International conference on intelligent robots and systems (pp. 919-924).

Niiyama, R., Nagakubo, A., \& Kuniyoshi, Y. (2007). Mowgli: a bipedal jumping and landing robot with an artificial musculoskeletal system. International Conference on Robotics and Automation, 7, 2546-2551.

Orrel, D., \& Smith, L. A. (2003). Visualizing bifurcations in high dimensional systems: the spectral bifurcation diagram. International Journal of Bifurcation and Chaos, 13(10), 3015-3027.

Pecora, L., \& Carroll, T. (1990). Synchronization in chaotic systems. Physical Review Letter, 64, 821.

Petersson, P., Granmo, M., \& Schouenborg, J. (2004). Properties of an adult spinal sensorimotor circuit shaped through early postnatal experience. Journal of Neurophysiology, 92, 280-288.

Pfeifer, R., Iida, F., \& Bongard, J. (2004). New robotics: design principles for intelligent systems. Artificial Life, Special Issue on New Robotics, Evolution and Embodied Cognition, 11(1-2), 99-120.

Pfeifer, R., Lungarella, M., \& Iida, F. (2007). Self-organization, embodiment, and biologically inspired robotics. Science, 318, 10881093.

Pikovsky, A., Rosenblum, M., Osipov, G., \& Kurths, J. (1997). Phase synchronization of chaotic oscillators by external driving. Physica $D, 104(3-4), 219-238$.

Pikovsky, A. S., Rosenblum, M. G., \& Kurths, J. (2001). Synchronization. Cambridge: Cambridge University Press.

Pitti, A., Lungarella, M., \& Kuniyoshi, Y. (2005). Quantification of emergent behaviors induced by feedback resonance of chaos. Recent Advances in Artificial Life: Advances in Natural Computation, 3(15), 199-213.

Pitti, A., Lungarella, M., \& Kuniyoshi, Y. (2006). Exploration of natural dynamics through resonance and chaos. In Proceedings of 9th conference on intelligent autonomous systems (pp. 558-565). 
Rabinovich, M., Varona, P., Selverston, A., \& Abarbanel, H. (2006). Dynamical principles in neuroscience. Reviews of Modern Physics, 78(1213), 1213-1265.

Rosenblum, M. G., Firsov, G. I., Kuuz, R. A., \& Pompe, B. (1998). Human postural control-force plate experiments and modelling. In H. Kantz, J. Kurths, \& G. Mayer-Kress (Eds.), Nonlinear analysis of physiological data (pp. 283-306). Berlin: Springer.

Rosenblum, M. G., Pikovsky, A. S., Kurths, J., Schaefer, C., \& Tass, P. (2001). Phase synchronization: from theory to data analysis. In F. Moss \& S. Gielen (Eds.), Neuro-informatics and neural modeling, series editor A. J. Hoff (pp. 279-321). Amsterdam: Elsevier. Chap. 9

Rosenblum, M. G., Pikovsky, A. S., \& Kurths, J. (1996). Phase synchronization of chaotic oscillators. Physical Review Letters, 76(11), 1804-1807.

Rummel, J., Iida, F., Smith, J., \& Seyfarth, A. (2008). Enlarging regions of stable running with segmented legs. In IEEE international conference on robotics and automation (ICRA) (pp. 367372). May 19-23, Pasadena, California.

Schaal, S. (2003). Movement planning and imitation by shaping nonlinear attractors. In Proceedings of the 12th Yale workshop on adaptive and learning systems.

Schouenborg, J. (2003). Somatosensory imprinting in spinal reflex modules. Journal of Rehabilitation Medicine. Supplement, 41, 73-80.

Selverston, A., Rabinovich, M., Abarbanel, H., Elson, R., Szucs, A., Pinto, R., Huerta, R., \& Varona, P. (2000). Reliable circuits from irregular neurons: a dynamical approach to understanding central pattern generators. Journal of Physiology, 94, 357-374.

Seo, K., \& Slotine, J. (2007). Models for global synchronization in CPG-based locomotion theory. In IEEE international conference on robotics and automation (pp. 281-286).

Seyfarth, A., Kalveram, K., \& Geyer, H. (2007). Simulating musclereflex dynamics in a simple hopping robot. In K. Berns \& $\mathrm{T}$. Luksch (Eds.), Autonome mobile systeme 2007 (pp. 294-300). Berlin: Springer.

Slotine, J., \& Lohmiller, W. (2001). Modularity, evolution, and the binding problem: a view from stability theory. Neural Networks, 14, 137-145.

Taga, G. (1994). Emergence of bipedal locomotion through entrainment among the neuro-musculo-skeletal system and the environment. Physica D, 75(1-3), 190-208.

Takuma, T., \& Hosoda, K. (2007). Terrain negotiation of a compliant biped robot driven by antagonistic artificial muscles. Journal of Robotics and Mechatronics, 19(4), 423-428.

Thelen, E., \& Smith, L. (1995). A dynamic systems approach to the development of cognition and action. Cambridge: MIT Press.

Ting, L. (2007). Dimensional reduction in sensorimotor systems: a framework for understanding muscle coordination of posture. Progress in Brain Research, 165, 299-321.

Ting, L., \& MacPherson, J. (2005). A limited set of muscle synergies for force control during a postural task. Journal of Neurophysiology, 93, 609-613.

Williamson, M. (1998). Exploiting natural dynamics in robot control. In Proceedings fourth European meeting on cybernetics and systems research (EMCSR '98) (pp. 1-6).

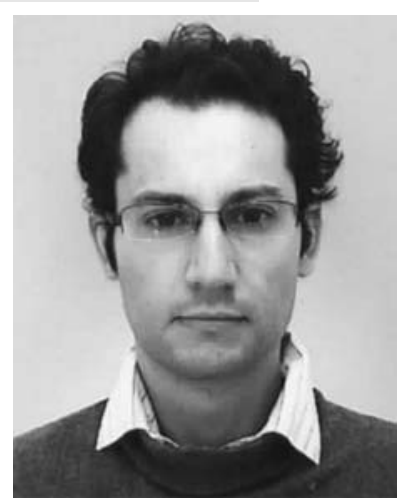

Alexandre Pitti received his M.Eng. degrees from Engineer School, ESME Sudria, Paris, France, and the University Pierre et Marie Curie, Paris, France, respectively, in 2002, and his Ph.D. degree from the University of Tokyo, Tokyo, Japan, in 2007. Currently, he is a Researcher at the ERATO Asada Synergistic Intelligence Project, JST. His research interests include complex systems, emergence and development of embodied cognition, human motion coordination and human action understanding.

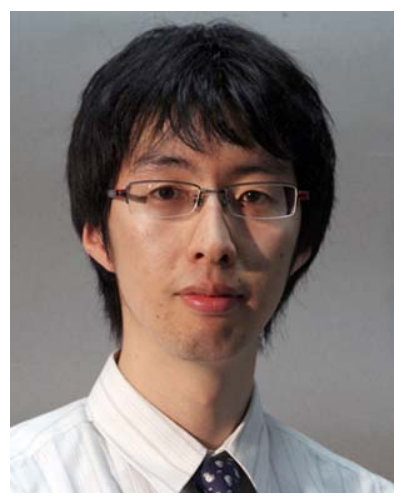

Ryuma Niiyama received the master's degree from the University of Tokyo, Tokyo, Japan, in 2007. Currently, he is a Ph.D. candidate at the Graduate School of Interdisciplinary Information Studies, the University of Tokyo. His current research interests are on the design and control of bio-inspired machines, especially musculo-skeletal robots.

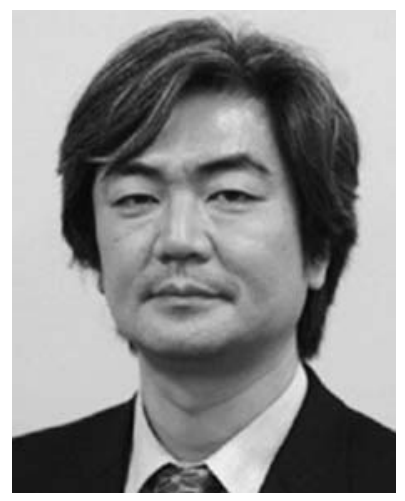

Yasuo Kuniyoshi received the M.Eng. and Ph.D. degrees from the University of Tokyo, Tokyo, Japan, in 1988 and 1991, respectively.

From 1991 to 2000, he was a Research Scientist and then a Senior Research Scientist at Electrotechnical Laboratory, AIST, MITI, Japan. From 1996 to 1997, he was a Visiting Scholar at Massachusetts Institute of Technology (MIT) AI Lab. Since 2001, he has been an Associate Professor at the University of Tokyo. Currently, he is a Professor at the Department of MechanoInformatics, School of Information Science and Technology, University of Tokyo. He is the author of over 200 technical publications, editorials, and books. His research interests include emergence and development of embodied cognition, human action understanding systems, and humanoid robots.

Dr. Kuniyoshi has received an Outstanding Paper Award from the International Joint Conference on Artificial Intelligence, Best Paper Award from the Robotics Society of Japan, Sato Memorial Award for Intelligent Robotics Research, and other awards. He is a member of the Robotics Society of Japan, Japan Society for Artificial Intelligence, Japanese Society of Baby Science, and other societies. 